\title{
Kernos
}

Revue internationale et pluridisciplinaire de religion grecque antique

19 | 2006

Varia

\section{Logiques catalogales et formes généalogiques}

Mythes grecs entre tradition orale et pratique de l'écriture

\section{Claude Calame}

\section{Q OpenEdition}

1 Journals

Édition électronique

URL : https://journals.openedition.org/kernos/424

DOI : 10.4000/kernos.424

ISSN : 2034-7871

Éditeur

Centre international d'étude de la religion grecque antique

Édition imprimée

Date de publication : 1 janvier 2006

Pagination : 23-29

ISSN : 0776-3824

\section{Référence électronique}

Claude Calame, "Logiques catalogales et formes généalogiques », Kernos [En ligne], 19| 2006, mis en ligne le 24 mai 2011, consulté le 24 août 2022. URL : http://journals.openedition.org/kernos/424 ; DOI : https://doi.org/10.4000/kernos.424

Tous droits réservés 


\section{Logiques catalogales et formes généalogiques : mythes grecs entre tradition orale et pratique de l'écriture}

\footnotetext{
Résumé : Attachée par Jack Goody à l'usage de l'écriture, la liste se révèle, dans une confrontation avec différentes traditions orales africaines, appartenir en fait à différentes formes de mémoire. Moyen mnémotechnique mis en forme selon différentes logiques d'ordre verbal autant que d'ordre sémantique, la liste devient alors, dans la mise en discours, un catalogue, avec sa pragmatique. Parmi ces logiques catalogales on compte la narration généalogique, privilégiée par les poètes animateurs de la mémoire de la communauté. Les logiques catalogales fournissent donc un instrument idéal pour penser les formes et les fonctions des genres mythologiques et mythographiques pratiquées par Grecs et Romains.
}

Abstract: Jack Goody has put into relation the list and the use of writing. Different types of oral tradition, especially in Western Africa, show that the list is actually a privileged form of memory. As such, the list is organised into different sequences according to different logic, on the verbal level as well as on the semantic one. Through these different formal organisations, the list becomes a catalogue with its pragmatics. Genealogical narration is one of these principles of discursive logic, often used by the poets, masters of the memory of the community. The logic of the catalogues is an ideal mechanism for thinking about the forms and genres of mythology and of mythography in Greece and Rome.

Dans sa tentative de définition d'une tradition écrite en contraste avec une tradition orale, Jack Goody attribue à la forme de la liste un rôle central. En effet les différentes formes d'inventaire (personnes, objets, événements) assumées par la liste seraient un critère de l'un des modes de pensée dépendant spécifiquement de l'usage de l'écriture. L'usage d'un système de notation graphique aurait une double fonction: d'une part enregistrer et archiver des données avec la possibilité de les communiquer à travers l'espace et dans le temps, d'autre part transposer ces données du domaine aural au domaine visuel, ce qui implique des possibilités de relecture dans des ordres différents, de reclassification et de décontextualisation. Par son caractère discontinu et sa logique distributive, la liste induirait un nouveau mode de connaissance; elle aurait un impact cognitif sur les modes de la pensée humaine. La liste s'inscrirait donc dans ces pratiques de l'écriture qui ont non seulement pour effet l'accumulation et l'augmentation des connaissances, mais aussi des modifications dans les représentations du monde. Que ce soit sous la forme de l'inventaire, du reçu, des comptes pour le payement d'un tribu ou pour la distribution de rations, de la séquence lexicale, de la chronique historique ou du «shopping list », la liste serait l'un des traits distinctifs de la «raison graphique ».

${ }^{1}$ J. Goody, The Domestication of the Savage Mind, Cambridge, University Press, 1977, p. 74 111 (dans un chapitre intitulé "What's in a list ?»). 


\section{Catalogues poétiques et inventaires oraux}

En ses différentes formes, la liste semble ainsi devoir s'inscrire dans le «Grand Partage » revisité par Jack Goody. En tant que résultat d'une pratique écrite, la liste paraît devoir constituer l'un des critères de la naissance de l'histoire, une histoire cumulative, rendue possible par l'archivage et les retours critiques que permet le document écrit.

Or dans un article d'une dizaine d'années postérieur au livre remarqué de Jack Goody, l'africaniste Christiane Seydou s'est appuyée sur les traditions orales des Peuls pour montrer qu'il existe plusieurs usages de la «fonctionliste » notamment dans la poésie; et qui plus est, dans la poésie de tradition orale! Elle fonde sa démonstration en particulier sur les listes de mots qui, chez les Peuls nomades de la boucle du Niger, peuvent assumer deux formes énumératives spécifiques; orales, ces formes poétiques de liste sont identifiées en tant que genres par une désignation particulière : soit que, dans la forme générique du mergol, la liste associe des mots sur une base phonétique dans un domaine technique tels un itinéraire de transhumance, une série de lieux d'abreuvage, une énumération d'instruments de pêche, ou autour d'un thème de sagesse tels la création du cosmos ou l'ordre du monde; soit que, dans la forme du jammooje na'i qui correspond à un éloge adressé aux bovins à l'occasion des fêtes de la transhumance, sont déclinées les qualités pastorales, mais aussi poétiques du peul accompli². Dans ces deux genres poétiques, Christiane Seydou identifie deux formes de liste: d'une part la forme du catalogue, par exemple de toponymes, dans une séquence d'éléments caractérisée par un trait sémantique commun; d'autre part le schéma de l'inventaire qui organise des objets hétérogènes dans un ensemble homogène. On ajoutera que la seconde forme semble devoir constituer un opérateur de classification.

Or la raison qui traverse et articule les catalogues aussi bien que les inventaires s'avère être souvent d'ordre poétique: raison phonique, rythmique, prosodique, mais aussi sémantique. Par anaphores, allitérations, échos rythmiques ou symétries phoniques interposés, le travail esthétique sur la substance sonore et sur les formes de l'expression y est particulièrement remarquable en concomitance avec le travail créatif sur la matière sémantique et sur les formes du contenu. En traversant et structurant les différentes formes de liste intégrés aux deux grands genres énumératifs mentionnés, cette raison poétique facilite non seulement l'apprentissage et la mémorisation des catalogues et inventaires oraux, mais elle en fonde également la «performance », la récitation rythmée.

Les différentes possibilités d'ordre et de logique visuels offertes par la mise en forme écrite d'une liste trouvent donc leur équivalent dans le travail sur la substance verbale et acoustique qui se révèle le propre des formes de liste intégrées aux genres poétiques fondant une tradition orale. Face aux jeux des accumulations et des retours critiques propres à la raison graphique il

${ }^{2}$ Ch. SEYDOU, « Raison poétique contre raison graphique », L'Homme 110 (1989), p. 50-68. 
convient d'être sensible aux différents rythmes imprimés au flux phonique dans la logique orale: travail sur le signifiant dans sa mesure rythmique (quantité) et dans sa couleur sonore (qualité) qui se double d'un travail poétique sur le signifié impliquant l'organisation générale des éléments inclus dans l'ensemble déployé par oral. Dans une tradition telle que la tradition poétique grecque classique où les pratiques de la communication et de la « performance » orales se mêlent peu à peu avec des formes discursives destinées à la lecture par le moyen de l'écriture alphabétique, les traits distinctifs de l'énumération orale peuvent se combiner avec ceux de l'inventaire écrit ${ }^{3}$. On pourra poser, de manière purement opératoire, que de simple liste l'énumération devient catalogue dès que la liste est soumise à une logique opérant sur les signifiants et sur les signifiés : catalogues dès lors poétiques, confiés à une tradition orale, puis à une tradition écrite, avec les mises en formes variées et les différentes logiques qu'impliquent ces différents modes de la communication, parfois en combinaison, avec leurs effets pragmatiques propres.

Les procédés énumératifs et les formes catalogales sont donc opératoires aussi bien dans des discours et une poésie de tradition orale que dans des pratiques discursives recourant à un système de notation graphique. Portant sur le signifiant verbal puis graphique, d'ordre aural puis visuel, ainsi que sur la matière sémantique dans toute son épaisseur, les logiques qui organisent les différentes formes de liste que sont les catalogues sont à l'évidence multiples. La systématisation de l'un ou l'autre de ces procédés de logique catalogale est à reconduire aux fonctions qu'assument ces différentes formes de discours; elle est à référer à leur destination pratique et par conséquent à leurs circonstances d'énonciation : fonctions pragmatiques d'efficacité de la mémoire, fonctions esthétiques de la communication et de la transmission.

Parmi ces formes discursives énumératives à forte composante pragmatique, la forme de la généalogie tient une place de choix. On rappellera simplement ici que, du point de vue discursif, la forme généalogique correspond à un procédé narratif cumulatif; par l'intermédiaire des deux figures de l'accouplement et de la procréation, le récit généalogique permet de déployer progressivement une série d'entités et d'états qualifiés pour conduire à un ensemble complexe et hiérarchisé; sa logique de l'arborescence dans la génération permet d'asserter par la narration autant un point d'origine que l'état complexe qui en résulte, après de nombreuses étapes de multiplication et de déploiement ${ }^{4}$. Et on ajoutera qu'en Grèce antique la forme du récit

\footnotetext{
3 Pour la poésie homérique qui se situe à l'intersection entre tradition orale et tradition écrite, on verra les exemples d'inventaires poétiques donnés par S. PERCEAU, La parole vive. Communiquer en catalogue dans l'épopée homérique, Louvain et al., Peeters, 2002, p. 49-72 et 156-184.

${ }^{4}$ Ch. JACOB, «L'ordre généalogique entre le mythe et l'histoire », in M. DETIENNE (éd.), Transcrire les mythologies. Tradition, écriture, bistoricité, Paris, Albin Michel, 1994, p. 169-202, a fort bien décrit les fonctions mémoriales des formes discursives grecques du catalogue et de la généalogie. J'ai tenté d'illustrer les procédures narratives du récit généalogique dans C. CALAME, «Le récit généalogique spartiate: la représentation mythologique d’une organisation spatiale »,
} 
généalogique a été largement systématisée et utilisée dans la reformulation du passé des cités selon une ligne chronologique unique; il s'agit là d'une pratique de l'écriture, d'une pratique historiographique qui s'appuie sur des formes poétiques relevant d'une tradition orale. Ce n'est pas un hasard si l'on a donné le titre de Genealogíai au traité d'historiographie grecque d'Hécatée; Hérodote ne désigne-t-il pas précisément comme "généalogiser » la réponse donnée par son prédécesseur et rival dans son enquête auprès des prêtres de Thèbes en Égypte ${ }^{5}$ ?

Sous forme de catalogue ou d'inventaire, la liste est donc un opérateur de création poétique et de mémoire. Dans la configuration des formes poétiques qui en assurent la communication, le mode de la tradition - orale ou écrite et le mode de la perception - acoustique ou visuelle - jouent sans doute un rôle important, mais secondaire. Le critère primordial de configuration est d'ordre pragmatique. C'est ce qu'illustre la brève comparaison que l'on aimerait proposer, à titre d'exemple, entre deux formes grecques de catalogue généalogique. Dans ces deux cas de mise en discours par le récit généalogique d'un catalogue de noms propres renvoyant à des figures divines et hérö̈ques, l'un relève du domaine communément considéré comme celui de la mythologie, l'autre appartient à celui de la mythographie.

\section{Catalogues généalogiques entre mythologie et mythographie}

Mais ensuite,

ayant couché avec Ciel, (Terre) enfanta Océan aux tourbillons profonds,

Coios, Crios, Hypérion et Japet,

Théia, Rhéia, Thémis et Mnémosyné,

Phoibé à la couronne d'or et Thétys la charmante;

le plus jeune après eux, vint au monde Cronos à l'esprit retors,

le plus terrible de ses enfants; il haïssait son père florissant.

Puis de Gé (Ouranos) a d'une part pour fils ceux que l'on nomme les Titans: Océan, Coios, Hypérion, Créios, Japet et le plus jeune de tous, Cronos; d'autre part il a pour filles celles que l'on appelle les Titanides: Thétys, Rhéa, Thémis, Mnémosyné, Phoibé, Dioné et Théia ${ }^{6}$.

QS 26 (1987), p. 43-91; voir maintenant P. BRULÉ, «Dans le nom, tout n'est-il pas déjà dit ? Histoire et géographie dans les récits généalogiques », Kernos 18 (2005), p. 241-268. Quant à l'impact du généalogique dans la figuration mythologique, voir L. COULOUBARITsIs, Aux origines de la philosophie européenne. De la pensée archaïque au néoplatonisme, Bruxelles, De Boeck, $1994^{2}$, p. 29-39.

5 Hérodote, II, 143, 1; voir à ce propos L. BerTELli, «Hecataeus: From Genealogy to Historiography ", in N. LuRAgHI (éd.), The Historian's Craft in the Age of Herodotus, Oxford, University Press, 2001, p. 67-94, en comparaison avec la tradition hésiodique et poétique du récit généalogique. Sur le rôle joué par la systématisation généalogique ou par la constitution d'une liste telle que celle des prêtresses d'Argos dans la construction historiographique d'une chronologie, les réflexions pertinentes de F. HARTOG, Evidence de l'histoire. Ce que voient les bistoriens, Paris, Éditions de l'EHESS, 2005, p. 45-61, sont encore dépendantes du partage opéré par Goody.

${ }^{6}$ Hésiode, Théogonie 132-138; Pseudo-Apollodore, Bibliothèque I, 1, 3. 
Pour cette mise en parallèle contrastive sont confrontés un extrait tiré du début de la Théogonie d'Hésiode et un passage appartenant au début de la Bibliothèque attribuée à Apollodore. D'un poème en hexamètres dactyliques composé probablement au début du $\mathrm{VII}^{\mathrm{e}}$ siècle avant notre ère pour une récitation orale par un aède on est ainsi passé à un traité en prose rédigé par écrit vers 200 de notre ère, sur un modèle remontant à l'époque hellénistique, et fort probablement destiné à un public de lecteurs ${ }^{7}$.

Dans ce passage, ce sont surtout les formes de l'expression qui ont subi les modifications les plus frappantes: substitution de la simple parataxe aux connecteurs d'énumération te que l'on trouve dans le texte hésiodique, élimination des épithètes homériques telles que khrusostéphanos ou erateinós, réorganisation des fils et des filles de Terre et de Ciel en deux listes homogènes (désormais distinguées et articulées par le connecteur de partition oppositive mén... dé), élimination des jeux d'assonances et des rimes internes typiquement hésiodiques (Kô̂ón te Krîon te..., v. 134; Theian te Rheian te..., v. 135, en même position métrique). Mais les formes du contenu n'échappent pas à la transformation puisqu'on assiste en particulier à une substitution particulièrement significative du point de vue du «gender » : Gé dans le récit généalogique de la Théogonie est remplacée par Ouranos dans la Bibliothèque comme sujet de l'action narrative. Par ailleurs, dans le texte attribué à Apollodore, Cronos est intégré par son simple nom propre, assorti d'une indication quant à son âge relatif, dans la liste des descendants masculins des deux dieux primordiaux alors que les deux vers que lui consacre le poème de la Théogonie annoncent le rôle déterminant qu'il est appelé à jouer dans le récit de la succession divine.

Il s'agit donc de différences essentiellement formelles, ou plus exactement de différences dans la léxis entendue comme «diction ». Du côté de la Théogonie d'Hésiode on aura reconnu les traits distinctifs de la diction poétique épique : articulation rythmique des énoncés en hexamètres dactyliques, couleur dialectale, épithètes qualifiantes, langage formulaire avec ses variations . Dans la Bibliothèque, on assiste, par les moyens d'une prose qui n'est plus métriquement rythmée, à des effets de pure liste : liste de noms propres dont la succession est réorganisée en deux séquences homogènes, par la réintégration de Cronos (le plus jeune des fils de Terrre et de Ciel) dans la liste des divinités masculines et par la réorganisation interne des deux séquences dans un ordre que l'on pourra supposer chronologique. S'il y a bien passage d'une diction poétique à une forme que nous identifions comme prosaïque, il est néanmoins difficile de reconduire les différences relevées à un passage d'une

\footnotetext{
${ }^{7}$ Pour les circonstances de composition et de communication de la Théogonie d'Hésiode, voir l'analyse comparative et les indications fournies par M.L. WEST, Hesiod. Theogony, Oxford, Clarendon Press, 1966, p. 1-16 et 40-48, ainsi que les indications bibliographiques données par C. CALAME, Le Récit en Grèce ancienne. Énonciations et représentations de poètes, Paris, Belin, 2000², p. 87-109; pour la Bibliothèque du Pseudo-Apollodore, cf. J.-C. CARRIÈre et B. MAssOniE, La Bibliothèque d'Apollodore, Besançon / Paris, Les Belles Lettres, 1991, p. 7-19.

${ }^{8}$ Sur la diction hésiodique, voir la synthèse proposée par WEST, o.c. (n. 7), p. 72-101
} 
organisation acoustique propre à une disposition visuelle autonome. Tracé en majuscules sur un rouleau de papyrus puis consigné en minuscules dans un cahier de parchemin, le texte de la Bibliothèque est soumis à la règle de la scriptio continua qui commande en Grèce à la transcription de toute forme de discours. On sait que, dans la représentation indigène classique, toute transcription à l'aide des caractères de l'alphabet emprunté au phénicien était conçue comme la visualisation rythmée du flux phonique; par conséquent la transcription graphique se réalise comme une écriture de la forme orale de l'énoncé. Le visuel ne fait donc que reproduire le rythme de l'aural. Si le rythme de la diction épique renvoie certainement à une récitation orale fondée sur la mémoire de l'aède, le flux scripturaire continu de la liste en prose pourrait relever d'une lecture à haute voix ${ }^{10}$. L'opposition entre poésie et prose ne peut donc être reconduite en ce qui concerne la Grèce antique à cet autre concept opératoire, chéri de la critique anthropologique d'inspiration structuraliste, qu'est l'opposition entre oral et écrit.

Mais, au-delà des différences relevées dans les formes de l'expression et de quelques aménagements dans les formes du contenu, le schéma d'organisation fondamental reste celui du récit généalogique dans la mise en intrigue de l'arborescence générative par l'intermédiaire de la figure de la procréation. Il revient en effet à la narration généalogique d'organiser de simples listes de noms propres en catalogues en introduisant notamment les marqueurs d'une argumentation narrative : le récit généalogique apparaît donc comme l'opérateur d'une « raison catalogale ». C'est dans la mise en intrigue généalogique que s'exerce le poieîn, le pouvoir créateur de l'aède homérique ou du compilateur hellénistique déployant de manière systématique le patrimoine mythologique de la culture grecque classique. Ce travail po(i)étique sur la matière phonique et sur la substance sémantique porte à s'interroger sur la pragmatique de ces différentes formes de catalogue généalogique : récit poétique justifiant pour une communauté de citoyens l'ordre divin sur lequel règne Zeus pour la Théogonie d'Hésiode ${ }^{11}$; manuel encyclopédique recueillant et

9 Cf. C. CALAME, «Rythme, voix et mémoire de l'écriture en Grèce ancienne », in R. PRETAGOSTINi (éd.), Tradizione e innovazione nella cultura greca da Omero all'età ellenistica. Scritti in onore di Bruno Gentili, Roma, GEI, 1993, I, p. 785-800. L’hypothèse développée à propos de la diction de Phérécyde de Syros par A. LAKs, «Écriture, prose, et les débuts de la philosophie grecque », Méthodos. Savoirs et textes 1 (2001), p. 131-151, montre qu'en fait l'équivalence oral / écrit $=$ poésie $/$ prose ne saurait être maintenue; je compte revenir sur cette impasse, discutée dans les échanges du colloque «Coralie» consacré en juin 2006 à l'EHESS à Paris à «La langue poétique des sages présocratiques ».

${ }^{10}$ Sur la fonction de réactualisation de la parole orale assumée par la lecture à haute voix, voir J. Svenbro, Phrasikleia. Anthropologie de la lecture en Grèce ancienne, Paris, La Découverte, 1988 , p. 53-73.

${ }^{11}$ Quelques-uns des usages poétiques de l'ordre généalogique qui articule le poème hésiodique précisément intitulé Catalogue des femmes font l'objet de la bonne étude comparative de G.B. D'AlEssio, «Ordered from the Catalogue: Pindar, Bacchylides, and Hesiodic genealogical poetry », in R. HunTER (éd.), The Hesiodic Catalogue of Women. Constructions and Reconstructions, Cambridge, University Press, 2005, p. 217-238; voir aussi R.L. FowLER, « Genealogical thinking, Hesiod's Catalogue, and the creation of the Hellenes», PCPh 44 (1998), p. 1-19. 
réorganisant par un travail de compilation l'ensemble du passé divin et héroïque de la Grèce pour un public de citadins en ce qui concerne la Bibliothèque d'Apollodore. Il ne s'agit là que d'une proposition, à discuter entre adeptes du CIERGA et membres du groupe « Polymnia ».

Quoi qu'il en soit, et sans faire un jeu de mots qui rappellerait par trop les suspectes ontologisations étymologisantes imposées par Heidegger, généalogie et catalogue sont à prendre au sens littéral qu'indique leur composition respective fondée sur lógos, un terme qui assumerait dans ce cas son double sens de «discours » et de « raison ». Raison discursive et raison pragmatique, l'organisation généalogique du catalogue garantit son efficacité dans des circonstances d'énonciation qui ne manquent pas d'avoir un impact sur la mise en discours du récit généalogique et sur son énoncé. À nous d'en déchiffrer les indices, à nous d'en restituer les finalités. Sans doute les logiques catalogales fournissent-elles un instrument privilégié pour penser non seulement les formes, mais surtout les rôles respectifs des genres mythologiques et des formes mythographiques pratiquées successivement par les Grecs et par les Romains. La pragmatique de ces logiques s'inscrit dans différents arts de la mémoire $^{12}$. Il s'agit d'en explorer les procédés et les fonctions.

Centre Louis Gernet

Claude CALAme

2, rue Vivienne

F - 75002 PARIS

Courriel : Claude.Calame@unil.ch

\footnotetext{
${ }^{12}$ À ce propos, du point de vue iconographique, voir par exemple C. SEvERI, Il percorso e la voce. Un'antropologia della memoria, Torino, Einaudi, 2004, p. 185-238.
} 